\title{
Parâmetros de qualidade da carne de cordeiros submetida aos processos de maturação e injeção de cloreto de cálcio
}

\author{
Quality parameters of aged and calcium chloride injected lamb meat
}

\author{
Nivea Maria Brancacci Lopes Zeola ${ }^{1}$ Pedro Alves de Souza ${ }^{2}$ \\ Hirasilva Borba Alves de Souza ${ }^{2}$ Américo Garcia da Silva Sobrinho ${ }^{3}$ \\ Elizabete Regina Leone Pelicano ${ }^{1}$
}

\section{RESUMO}

Foram estudados o pH e a temperatura dos músculos Biceps femoris, Longissimus e Triceps brachii de cordeiros "Morada Nova", durante o período de instalação do rigor mortis, e os efeitos da maturação e da injeção de cloreto de cálcio nas características qualitativas destes músculos. Os cordeiros foram abatidos ao atingirem $25 \mathrm{~kg}$ de peso vivo. O declínio do pH e da temperatura dos músculos foram monitorados durante a instalação do rigor mortis nos tempos 0, 2, 4, 6, 8, 10, 12 e 24 horas após o abate, com as carcaças refrigeradas em câmara fria $a 4^{\circ} \mathrm{C}$. Após este período, os músculos foram retirados e submetidos aos respectivos tratamentos com posterior determinação da perda de peso durante a cocção e da maciez. A queda do pH para os músculos Biceps femoris e Longissimus mostrou-se dentro da faixa considerada normal para carne ovina, enquanto o músculo Triceps brachii apresentou pH, às 10,12 e 24 horas, considerado acima da faixa descrita. O declínio da temperatura apresentouse típico de condições de resfriamento habituais. O tempo de maturação e a injeção de cloreto de cálcio não afetaram a perda de peso durante a cocção. Em relação à maciez, o tempo de maturação nos músculos Biceps femoris e Longissimus propiciou carne mais tenra e a concentração de 0,3M de cloreto de cálcio melhorou a maciez do músculo Longissimus.

Palavras-chave: cordeiros, cloreto de cálcio, maturação, $p H$, temperatura.

\section{ABSTRACT}

Temperature and $\mathrm{pH}$ of Biceps femoris, Longissimus and Triceps brachii muscles from 'Morada Nova' lambs during the rigor mortis and the effects of ageing and calcium chloride injection in qualitative characteristics of these muscles were studied. The lambs were slaughtered with $25 \mathrm{~kg}$ live weight. The decline of $\mathrm{pH}$ and temperature of muscles were monitored during the onset of rigor mortis in times $0,2,4,6,8$,
10, 12 and 24 hours after slaughter, with the carcasses refrigerated at $4^{\circ} \mathrm{C}$. The muscles were removed and submitted to respective treatments with determination of cooking loss and tenderness. The decline of $\mathrm{pH}$ from Biceps femoris and Longissimus was tipical normal processs, while Triceps brachii presented $\mathrm{pH}$ at 10, 12 and 24 hours considered higher. The decline of temperature was tipical from normal process. The ageing and calcium chloride injection did not affect cooking loss. The ageing in Biceps femoris and Longissimus improved the tenderness while the 0.3M calcium chloride injection only improved tenderness in Longissimus.

Key words: lambs, calcium chloride, ageing, $p H$, temperature.

\section{INTRODUÇÃO}

De acordo com o Anuário da Pecuária Brasileira, o rebanho ovino do Brasil distribui-se em 10.129.267 de cabeças na região Nordeste, 4.691.472 na região Sul, 1.051.739 na região Centro-Oeste, 678.991 na região Sudeste e 554.103 na região Norte, sendo que, no ano de 2005, foram abatidos legalmente apenas 909.699 cabeças entre ovinos e caprinos (ANUALPEC, 2006). O consumo de carne ovina no Brasil ainda é baixo, em torno de 800g/per capita/ano; no entanto, o setor está em fase de crescimento. Estima-se que a produção atingirá 100 milhões de cabeças nos próximos 10 anos e o segmento irá se firmar como boa alternativa de aplicação, sendo que atualmente a criação de cordeiros no País movimenta R\$ 360 milhões ao ano, considerando o preço de venda do quilo da carne a R\$5,00 (GALVÃO, 2004).

${ }^{1}$ Curso de Pós-graduação em Zootecnia, Universidade Estadual Paulista, Faculdade de Ciência Agrárias e Veterinárias (Unesp/ FCAV), Departamento de Tecnologia. Via de Acesso Prof. Paulo Donato Castellane, s/n, 14884-900, Jaboticabal, SP, Brasil. Email: dzeola@ig.com.br. Autor para correspondência.

${ }^{2}$ Departamento de Tecnologia, FCAV/Unesp, Jaboticabal, SP, Brasil.

${ }^{3}$ Departamento de Zootecnia, FCAV/Unesp, Jaboticabal, SP, Brasil. 
As raças de corte utilizadas tradicionalmente pelos produtores no Estado de São Paulo, como "Ile de France” e "Suffolk” estão sendo substituídas pelas raças deslanadas (CUNHA et al., 2004). No entanto, estudos a respeito da qualidade da carne de cordeiros “Morada Nova” são escassos. Atualmente, o mercado consumidor apresenta elevada exigência em relação às características qualitativas da carne, tornando necessário o conhecimento de parâmetros de qualidade no sistema de produção de ovinos destinados ao abate (BRESSAN et al., 2001).

Desta forma, estudos sobre o processo da instalação do rigor mortis, pelas averiguações do $\mathrm{pH}$ e da temperatura da carcaça durante o resfriamento, e sobre técnicas utilizadas pós-abate, como a utilização do processo de maturação e do cloreto de cálcio, apresentam contribuição relevante para a pesquisa (BAWCOM et al., 1995). Como a atividade de calpaína é pouco ativada em condições normais post rigor mortem, a utilização de injeção de solução a base de cloreto de cálcio auxilia a ativação das proteases cálciodependentes, como a m-calpaína, e de sua inibidora, a calpastatina, induzindo à tenderização da carne (MOURA et al., 1999). No entanto, os artigos encontrados na literatura a respeito da qualidade da carne de cordeiros "Morada Nova” são escassos. Também raros, senão inexistentes, são os trabalhos que estudaram o tempo ideal de maturação e a utilização do cloreto de cálcio nas carnes provenientes desta raça.

Para que o músculo de um animal abatido se transforme em carne, é necessário que ocorram processos bioquímicos conhecidos como modificações post mortem. Dentre estes, ocorre alteração do $\mathrm{pH}$, que pode chegar a 5,4, duas a oito horas após a sangria. De acordo com PRATES (2000), a carne ovina atinge $\mathrm{pH}$ final entre 5,5 a 5,8 de 12 a 24 horas após o abate.

O estresse do animal por período prolongado ou intenso exercício muscular no pré-abate causam redução nos níveis de glicogênio, produzindo carnes DFD (dark, firm and dry), ou seja, exibem $\mathrm{pH}$ alto, coloração escura e textura firme, sendo mais secas, porém, como têm alta capacidade de retenção de água, quando cozidas são freqüentemente suculentas. Já as carnes PSE (pale, soft and exudative) são oriundas de animais submetidos ao estresse e imediatamente abatidos. Assim, o pH decresce rapidamente, conferindo carnes pálidas, moles e exudativas, com baixa capacidade de retenção de água. (MILLER, 2001).

Sendo assim, este trabalho foi planejado com o objetivo de estudar o $\mathrm{pH}$ e a temperatura dos músculos Biceps femoris (patinho), Longissimus e Triceps brachii (coração da paleta) de cordeiros Morada Nova durante o período de instalação do rigor mortis e avaliar os efeitos da utilização de diferentes períodos de maturação e da injeção de cloreto de cálcio 0,3M nas características qualitativas destes músculos.

\section{MATERIAL E MÉTODOS}

Foram utilizados 24 cordeiros da raça “Morada Nova”, machos inteiros, com peso vivo médio inicial de $15 \mathrm{~kg}$, os quais permaneceram alojados em galpão de confinamento até o abate, em baias coletivas de $12 \mathrm{~m}^{2}$ cada, compostas por seis cordeiros, totalizando quatro baias equipadas com comedouros e bebedouros. A dieta experimental formulada de acordo com o AFRC (1995) teve como volumoso feno de Coast cross moído, e, como concentrado, farelo de soja e milho moído, além de sal iodado e núcleo mineral ovino.

Os cordeiros foram abatidos ao atingirem $25 \mathrm{~kg}$ de peso vivo. O abate foi realizado após jejum de 16 horas de dietas hídrica e sólida. Após este período, os cordeiros foram insensibilizados por meio de descarga elétrica de $220 \mathrm{~V}$ por 8 segundos, e, em seguida, foram seccionadas as veias jugulares e as artérias carótidas para a sangria. Imediatamente após o abate, nos músculos Biceps femoris, Longissimus e Triceps brachii, foram determinados o $\mathrm{pH}$ e a temperatura. A leitura do $\mathrm{pH}$ foi feita com a utilização de eletrodo de penetração e da temperatura por meio de termômetro digital. O declínio do pH e da temperatura dos músculos foram monitorados durante a instalação do rigor mortis nos tempos 0, 2, 4, 6, 8, 10, 12 e 24 horas após o abate, com as carcaças refrigeradas em câmara fria a $4^{\circ} \mathrm{C}$.

Após este período, os músculos (do lado esquerdo da carcaça) Biceps femoris (BF), Longissimus (L) e Triceps brachii (TB) foram retirados para serem submetidos aos tratamentos: controle; maturação durante 7 dias; maturação durante 14 dias; injeção de cloreto de cálcio 0,3M; injeção de cloreto de cálcio 0,3M e maturação durante sete dias e injeção de cloreto de cálcio 0,3M e maturação durante 14 dias. A solução de cloreto de cálcio foi preparada com água destilada e injetada na carne na proporção de $10 \%$ do peso da peça (JATURASITHA et al., 2004). Posteriormente foram determinadas a perda de peso durante a cocção e a maciez (OSÓRIO et al., 1998).

O delineamento experimental utilizado para as medidas de $\mathrm{pH}$ e de temperatura foi em blocos casualizados, com três tratamentos (músculos Biceps femoris, Longissimus e Triceps brachii) e seis repetições em cada bloco. O delineamento experimental utilizado para as determinações da perda de peso durante a cocção e maciez foi em blocos casualizados, em esquema fatorial $2 \times 3$, com seis tratamentos e quatro blocos, sendo os fatores avaliados: a injeção de cloreto 
de cálcio - $\mathrm{CaCl}_{2}, 10 \%$ do peso da peça ( 0 e $\left.0,3 \mathrm{M}\right)$ e o tempo de maturação (0, 7 e 14 dias). Todas as análises de variância foram realizadas de acordo com o SAS (1996) e as comparações dos contrastes entre as médias dos tratamentos foram feitas pelo teste de Tukey a 5\%.

\section{RESULTADOS E DISCUSSÃO}

Os dados contidos na tabela 1 permitem observar que, entre os tempos 2, 6 e 8 horas após o abate, não houve diferença significativa $(\mathrm{P}>0,05)$ para os valores de $\mathrm{pH}$ entre os músculos estudados, com valores médios de 6,42; 6,11 e 6,01, respectivamente. Em contrapartida, PEREZ et al. (1997), avaliando a queda do $\mathrm{pH}$ na carne ovina, verificaram diferença significativa $(\mathrm{P}<0,05)$ no tempo 6 horas para os músculos Biceps femoris e Longissimus dorsi, com maior valor de $\mathrm{pH}$ para o Biceps femoris.

Para os tempos 0, 4, 10, 12 e 24 horas após o abate, houve diferença significativa $(\mathrm{P}<0,05)$ para os valores de $\mathrm{pH}$ entre os músculos estudados. No tempos 0 e 4 horas, o músculo BF apresentou maior $\mathrm{pH}$ em relação aos músculos L e TB. Entretanto, nos tempos 10, 12 e 24 horas, o $\mathrm{pH}$ mais elevado foi apresentado pelo músculo TB, com valores de 6,02; 5,96 e 5,93, respectivamente.

A faixa de $\mathrm{pH}$ considerada normal 24 horas após o abate é de 5,5 a 5,8 (PARDI et al., 2001). Neste experimento, os músculos BF e L apresentaram valores de $\mathrm{pH}$ neste intervalo, embora o mesmo não tenha ocorrido com o músculo TB, que apresentou $\mathrm{pH}$ superior ao citado acima.

A figura 1 representa o gráfico contendo as equações de regressão entre o valor de pH e o horário de medição. ROTA(2005), avaliando o pH 0 e 24 horas após o abate no músculo Longissimus dorsi de cordeiros Corriedale, obteve valor superior para o $\mathrm{pH} 0$ horas $(6,72)$ em comparação ao obtido neste estudo $(6,47)$, entretanto, para o $\mathrm{pH} 24$ horas, obteve valor inferior $(5,54)$ ao apresentado nesta pesquisa $(5,73)$.

Em estudo para averiguar a queda do $\mathrm{pH}$ nos músculos Longissimus dorsi e Triceps brachii de cordeiros Santa Inês, OLIVEIRA et al. (2003) observaram que não houve diferença significativa $(\mathrm{P}>0,05)$ entre $\mathrm{o}$ $\mathrm{pH}$ dos músculos medido nos tempos 0 e 24 horas. $\mathrm{O}$ pH dos músculos no tempo 0 horas foi de 6,67 e 6,73 e no tempo 24 horas foi de 5,61 e 5,68 para o Longissimus e para o Triceps brachii, respectivamente. Entretanto, este fato não ocorreu neste experimento, pois neste caso, nos tempos 0 e 24 horas, houve diferença significativa $(\mathrm{P}<0,05)$ entre os valores de $\mathrm{pH}$ dos músculos.

Os dados elucidados na tabela 1 permitem observar que, nos tempos 0 e 24 horas após o abate, não houve diferença significativa $(\mathrm{P}>0,05)$ para os valores de temperatura entre os músculos estudados, com valores médios de 35,04 e de $4,15^{\circ} \mathrm{C}$, respectivamente. Entretanto, este fato não ocorreu para os tempos 2, 4, 6, 8, 10 e 12 horas após o abate, nos quais houve diferença significativa $(\mathrm{P}<0,05)$ para os valores de temperatura entre os músculos estudados. Com exceção do tempo 8 horas, nos demais tempos a maior temperatura observada foi no músculo BF.

A figura 2 representa o gráfico contendo as equações de regressão entre o valor de temperatura e o horário de medição. O declínio da temperatura apresentou comportamento exponencial, indicando uma rápida queda nas primeiras horas post mortem seguida

Tabela 1 - Medidas de pH e temperatura dos músculos Biceps femoris (BF), Longissimus (L) e Triceps brachii (TB) de cordeiros da raça "Morada Nova" durante a instalação do rigor mortis.

\begin{tabular}{|c|c|c|c|c|c|c|c|c|}
\hline \multirow{3}{*}{ Músculo } & \multicolumn{8}{|c|}{$\mathrm{pH}$} \\
\hline & \multicolumn{8}{|c|}{ Tempo após o abate (horas) } \\
\hline & 0 & 2 & 4 & 6 & 8 & 10 & 12 & 24 \\
\hline $\mathrm{BF}$ & $6,63^{\mathrm{a}}$ & 6,48 & $6,34^{\mathrm{a}}$ & 6,15 & 6,01 & $5,91^{\mathrm{b}}$ & $5,81^{\mathrm{b}}$ & $5,75^{\mathrm{b}}$ \\
\hline $\mathrm{L}$ & $6,47^{\mathrm{b}}$ & 6,41 & $6,15^{\mathrm{b}}$ & 6,06 & 5,95 & $5,89^{b}$ & $5,81^{b}$ & $5,73^{b}$ \\
\hline $\mathrm{TB}$ & $6,39^{c}$ & 6,36 & $6,16^{\mathrm{b}}$ & 6,12 & 6,06 & $6,02^{\mathrm{a}}$ & $5,96^{\mathrm{a}}$ & $5,93^{\mathrm{a}}$ \\
\hline \multirow[t]{2}{*}{ CV (\%) } & 0,56 & 0,91 & 1,19 & 0,93 & 0,97 & 0,66 & 0,56 & 0,63 \\
\hline & \multicolumn{8}{|c|}{ Temperatura $\left({ }^{\circ} \mathrm{C}\right)$} \\
\hline $\mathrm{BF}$ & 35,03 & $15,43^{\mathrm{a}}$ & $8,09^{a}$ & $6,78^{\mathrm{a}}$ & $5,47^{\mathrm{a}}$ & $5,43^{\mathrm{a}}$ & $4,92^{\mathrm{a}}$ & 4,62 \\
\hline $\mathrm{L}$ & 35,10 & $12,29^{b}$ & $5,90^{\mathrm{b}}$ & $4,71^{\mathrm{b}}$ & $4,56^{\mathrm{ab}}$ & $4,16^{\mathrm{b}}$ & $3,86^{\mathrm{b}}$ & 3,85 \\
\hline TB & 34,99 & $12,34^{\mathrm{b}}$ & $6,27^{\mathrm{b}}$ & $5,24^{\mathrm{b}}$ & $4,41^{\mathrm{b}}$ & $4,07^{\mathrm{b}}$ & $4,04^{\mathrm{b}}$ & 3,97 \\
\hline CV (\%) & 1,12 & 7,35 & 5,69 & 10,15 & 9,77 & 7,22 & 6,77 & 8,98 \\
\hline
\end{tabular}

a,b,c Letras distintas na mesma coluna diferem pelo teste de Tukey a 5\% de probabilidade; CV - coeficiente de variação. 


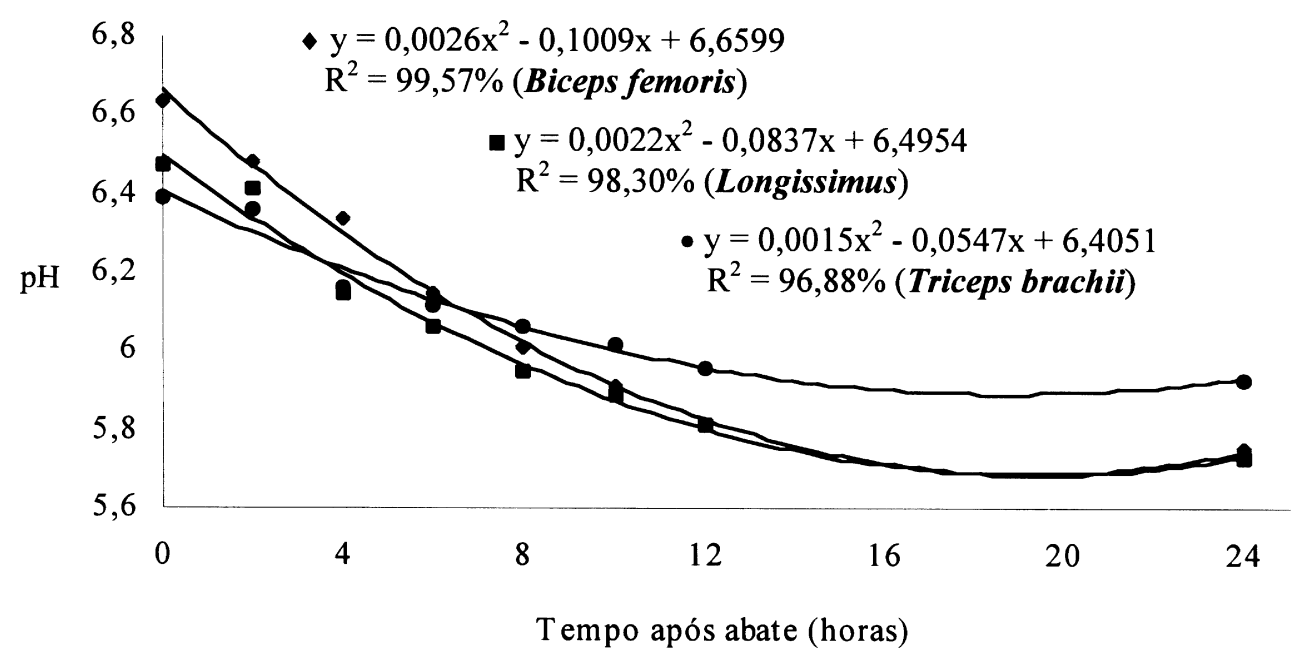

Figura 1 - Curva de queda do pH nos músculos Biceps femoris, Longissimus e Triceps brachii de cordeiros da raça "Morada Nova” durante a instalação do rigor mortis.

de estabilização. O coeficiente de determinação $\left(\mathrm{R}^{2}\right)$ indicou ajustamento eficiente dos dados em torno da curva de regressão.

OLIVEIRA et al. (2003), estudando a queda da temperatura nos músculos Longissimus dorsi e Triceps brachii de cordeiros Santa Inês, observaram valores de $28,58^{\circ} \mathrm{C}$ para o tempo 0 horas, $6,26^{\circ} \mathrm{C}$ para o tempo 8 horas e $0,24^{\circ} \mathrm{C}$ para o tempo 24 horas após o abate. Estes dados diferem dos obtidos nesta pesquisa, pois, nos tempos 0 e 24 horas, a temperatura da carcaça foi maior, enquanto que no tempo 8 horas, foi menor.

Conforme os valores da tabela 2, pode-se observar que o tempo de maturação não influenciou ( $\mathrm{P}>0,05)$ a perda de peso durante a cocção dos músculos BF, Le TB, com valores médios de 34,66; 30,50 e 34,59\%, respectivamente. Entretanto, SAÑUDO (2002) citou

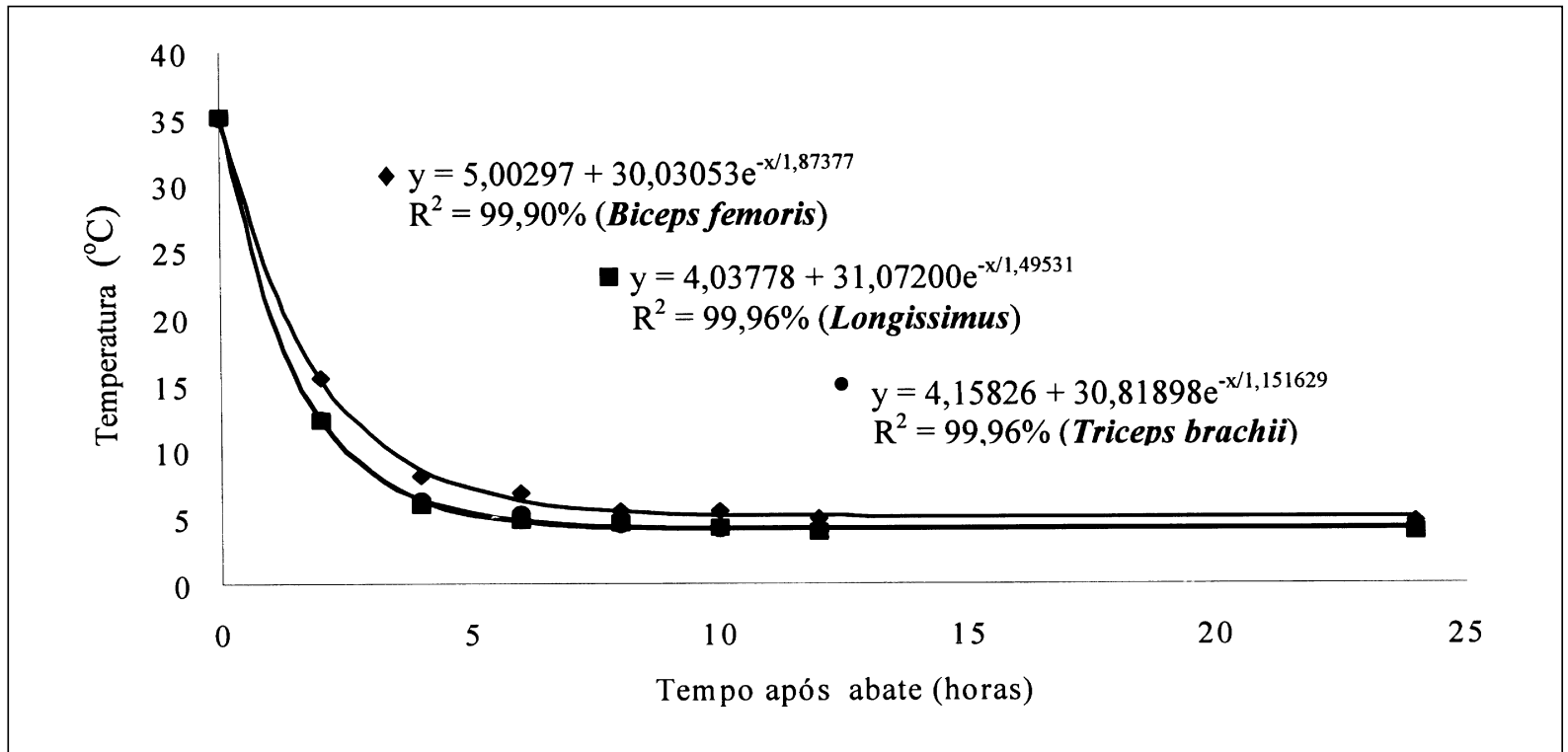

Figura 2 - Curva de queda da temperatura nos músculos Biceps femoris, Longissimus e Triceps brachii de cordeiros da raça "Morada Nova” durante a instalação do rigor mortis.

Ciência Rural, v.36, n.5, set-out, 2006. 
Tabela 2 - Valores de perda de peso durante a cocção (PPC) das carnes provenientes dos músculos Biceps femoris (BF), Longissimus (L) e Triceps brachii (TB) em cordeiros da raça "Morada Nova” nos diferentes tempos de maturação e nas diferentes concentrações de cloreto de cálcio.

\begin{tabular}{|c|c|c|c|}
\hline \multirow{2}{*}{ Tempo de maturação (M) } & \multicolumn{3}{|c|}{ PPC (\%) } \\
\hline & $\mathrm{BF}$ & $\mathrm{L}$ & TB \\
\hline Sem maturação $^{1}$ & 35,57 & 33,18 & 34,55 \\
\hline $7 \operatorname{dias}^{2}$ & 35,50 & 29,11 & 34,75 \\
\hline $14 \operatorname{dias}^{3}$ & 32,90 & 29,22 & 34,46 \\
\hline Teste F & 0,66 & 1,56 & 0,01 \\
\hline $\mathrm{P}$ & 0,5331 & 0,2423 & 0,9913 \\
\hline DMS & 6,89 & 6,67 & 5,83 \\
\hline \multicolumn{4}{|c|}{ Concentração de cloreto de cálcio (C) } \\
\hline Sem cloreto de cálcio ${ }^{4}$ & 33,54 & 29,46 & $31,46^{\mathrm{b}}$ \\
\hline $0,3 \mathrm{M}^{5}$ & 35,77 & 31,55 & $37,72^{\mathrm{a}}$ \\
\hline Teste F & 1,06 & 0,96 & $11,69 * *$ \\
\hline $\mathrm{P}$ & 0,3203 & 0,3427 & 0,0038 \\
\hline DMS & 4,62 & 4,57 & 3,91 \\
\hline Teste F para int. M x C & 0,58 & 0,61 & 0,58 \\
\hline $\mathrm{P}$ & 0,5744 & 0,5561 & 0,5732 \\
\hline CV(\%) & 15,31 & 17,20 & 12,98 \\
\hline
\end{tabular}

${ }^{\text {a,b }}$ Dentro de um mesmo fator, médias seguidas por letras distintas na coluna diferem entre si pelo teste de Tukey. DMS - diferença mínima significativa. P - probabilidade. CV - coeficiente de variação. ${ }^{1}$ Carne sem maturação; ${ }^{2}$ Carne maturada por 7 dias; ${ }^{3}$ Carne maturada por 14 dias; ${ }^{4}$ Carne sem injeção de cloreto de cálcio; ${ }^{5}$ Carne injetada com cloreto de cálcio 0,3M.

que, conforme aumenta o tempo de maturação, as perdas de água tendem a ser maiores. Sendo assim, ZAPATA et al. (2003) observaram no músculo Longissimus dorsi de cordeiros que, conforme aumentou o tempo de maturação, a perda de peso durante a cocção foi maior; para as carnes não maturadas, a perda foi de 26,96\%; já para as carnes maturadas por 21 dias, a perda foi de $34,92 \%$.

A injeção de cloreto de cálcio não afetou $(\mathrm{P}>0,05)$ a perda de peso durante a cocção dos músculos BF e L, com valores médios de 34,66 e 30,51\%, respectivamente. Em contrapartida, influenciou $(\mathrm{P}<0,05)$ a perda de peso durante a cocção do músculo $\mathrm{TB}$, com maior perda $(37,72 \%)$ para $0,3 \mathrm{M}$ de $\mathrm{CaCl}_{2}$. Corroborando com este fato, KOOHMARAIE et al. (1990) também observaram maior perda de peso durante a cocção $(26,1 \%)$ nas carnes tratadas com $\mathrm{CaCl}_{2}$ em comparação às do grupo controle (24,6\%), apesar de o músculo avaliado ter sido o Longissimus dorsi. A interação entre os fatores estudados não foi significativa $(\mathrm{P}>0,05)$.

Na tabela 3, observa-se que o tempo de maturação não influenciou $(\mathrm{P}>0,05)$ a maciez do músculo TB, com valor médio de $1,70 \mathrm{kgf} / \mathrm{cm}^{2}$. Entretanto, influenciou $(\mathrm{P}<0,05)$ a maciez dos músculos BF e L. No músculo BF, a maciez foi maior nas carnes maturadas durante 7 (2,20kgf cm$\left.{ }^{-2}\right)$ e 14 dias $(1,76 \mathrm{kgf}$ $\left.\mathrm{cm}^{-2}\right)$, as quais não diferiram entre si $(\mathrm{P}>0,05)$. Para 0 músculo L, o processo foi o mesmo, observando-se melhores valores de maciez para carnes maturadas durante $7\left(2,21 \mathrm{kgf} \mathrm{cm}^{-2}\right)$ e 14 dias $\left(1,89 \mathrm{kgf} \mathrm{cm}^{-2}\right)$, as quais também não diferiram entre si $(\mathrm{P}>0,05)$. Do mesmo modo, em estudo para avaliar o efeito da maturação na carne ovina, KOOHMARAIE et al. (1990) observaram redução na força de cisalhamento para carne maturada durante 1 e 7 dias, com valores de 8,9 e 5,4kgf, respectivamente.

A injeção de cloreto de cálcio não afetou $(\mathrm{P}>0,05)$ a maciez dos músculos $\mathrm{BF}$ e TB, com valores médios de 2,24 e 1,71 kgf $\mathrm{cm}^{-2}$, respectivamente. Entretanto, afetou $(\mathrm{P}<0,05)$ a maciez do músculo L. As carnes injetadas com $0,3 \mathrm{M} \mathrm{de} \mathrm{CaCl}_{2}$ foram mais macias $\left(1,86 \mathrm{kgf} \mathrm{cm}^{-2}\right)$ quando comparadas às que não foram injetadas $\left(2,89 \mathrm{kgf} \mathrm{cm}^{-2}\right)$. Da mesma forma, ao avaliarem o efeito da injeção de cloreto de cálcio em carcaças de cordeiros, WHEELER et al. (1991) concluíram que as carnes do grupo controle apresentaram maior força de 
Tabela 3 - Valores de força de cisalhamento (FC) das carnes provenientes dos músculos Biceps femoris (BF), Longissimus (L) e Triceps brachii (TB) em cordeiros da raça "Morada Nova” nos diferentes tempos de maturação e nas diferentes concentrações de cloreto de cálcio.

\begin{tabular}{|c|c|c|c|}
\hline \multirow{2}{*}{ Tempo de maturação (M) } & \multicolumn{3}{|c|}{$\mathrm{FC}\left(\mathrm{kgf} / \mathrm{cm}^{2}\right)$} \\
\hline & $\mathrm{BF}$ & $\mathrm{L}$ & ТВ \\
\hline Sem maturação ${ }^{1}$ & $2,76^{\mathrm{a}}$ & $3,03^{\mathrm{a}}$ & 1,96 \\
\hline $7 \operatorname{dias}^{2}$ & $2,20^{\mathrm{ab}}$ & $2,21^{\mathrm{b}}$ & 1,85 \\
\hline $14 \operatorname{dias}^{3}$ & $1,76^{\mathrm{b}}$ & $1,89^{\mathrm{b}}$ & 1,30 \\
\hline Teste F & $6,00 *$ & $4,16 *$ & 3,60 \\
\hline $\mathrm{P}$ & 0,0122 & 0,0366 & 0,0527 \\
\hline DMS & 0,75 & 1,06 & 0,6811 \\
\hline \multicolumn{4}{|c|}{ Concentração de cloreto de cálcio (C) } \\
\hline Sem cloreto de cálcio ${ }^{4}$ & 2,29 & $2,89^{\mathrm{a}}$ & 1,91 \\
\hline $0,3 \mathbf{M}^{5}$ & 2,19 & $1,86^{\mathrm{b}}$ & 1,50 \\
\hline Teste F & 0,20 & $9,61^{* *}$ & 3,77 \\
\hline $\mathrm{P}$ & 0,6612 & 0,0073 & 0,0711 \\
\hline DMS & 0,50 & 0,71 & 0,46 \\
\hline Teste F para int. M x C & 1,80 & 0,10 & 0,01 \\
\hline $\mathrm{P}$ & 0,1985 & 0,9040 & 0,9928 \\
\hline $\mathrm{CV}(\%)$ & 25,88 & 34,30 & 30,80 \\
\hline
\end{tabular}

${ }^{\text {a,b }}$ Dentro de um mesmo fator, médias seguidas por letras distintas na coluna, diferem entre si pelo teste de Tukey. DMS - diferença mínima significativa. P - probabilidade. CV - coeficiente de variação. ${ }^{1}$ Carne sem maturação; ${ }^{2}$ Carne maturada por 7 dias; ${ }^{3}$ Carne maturada por 14 dias; ${ }^{4}$ Carne sem injeção de cloreto de cálcio; ${ }^{5}$ Carne injetada com cloreto de cálcio 0,3M.

cisalhamento (3,14kgf) em comparação às carnes do grupo que recebeu injeção de cloreto de cálcio (2,67kgf). Entre os fatores estudados não houve interação significativa $(\mathrm{P}>0,05)$.

\section{CONCLUSÃO}

A queda do $\mathrm{pH}$ no Biceps femoris e no Longissimus está adequada para a carne ovina, sendo o declínio da temperatura típico de condições de resfriamento habituais. A maturação influi sobre a maciez do Biceps femoris e do Longissimus, enquanto o cloreto de cálcio influi sobre a perda de peso durante a cocção do Triceps brachii e sobre a maciez do Longissimus.

\section{AGRADECIMENTOS}

Ao Conselho Nacional de Desenvolvimento Científico e Tecnológico (CNPq), pela concessão da bolsa de estudos; ao Programa de Pós-graduação em Zootecnia da Faculdade de Ciências Agrárias e Veterinárias (FCAV), Universidade Estadual Paulista (Unesp) e à Fundação para o Desenvolvimento da Universidade Estadual Paulista Júlio de Mesquita Filho (FUNDUNESP), pelo recurso financeiro.

\section{REFERÊNCIAS}

AGRICULTURAL AND FOOD RESEARCH COUNCIL. Energy and protein requirements of ruminants. Washington: CAB International, 1995. 159p.

ANUÁRIO DA PECUÁRIA BRASILEIRA - Anualpec. São Paulo, 2006. p.277.

BAWCOM, D.W. et al. Reduction of microorganisms on beef surfaces utilizing electricity. Journal of Food Protection, Des Moines, v.58, n.1, p.35-38, 1995.

BRESSAN, C. et al. Efeito do peso ao abate de cordeiros Santa Inês e Bergamácia sobre as características físico-químicas da carne. Ciência e Tecnologia de Alimentos, Campinas, v.21, n.3, p.293-303, 2001.

CUNHA, E.A. et al. Carcaças de cordeiros de raças de corte em criação intensiva. O Berro, Uberaba, n.68, p.46-50, 2004.

GALVÃO, F. Rebanho lucrativo. Isto é Dinheiro, São Paulo, n.350, p.82-83, 2004.

JATURASITHA, S. et al. Reducing toughness of beef from Bos indicus draught steers by injection of calcium chloride. Effect of concentration and time post mortem. Meat Science, Barking, v.68, n.1, p.61-69, 2004.

KOOHMARAIE, M. et al. Acceleration of post mortem 
tenderization in lamb and Brahman-cross beef carcasses through infusion of calcium chloride. Journal of Animal Science, Champaign, v.68, n.5, p.1278-1283, 1990.

MILLER, R.K. Obtendo carne de qualidade consistente. In: CONGRESSO BRASILEIRO DE CIÊNCIA E TECNOLOGIA DE CARNES, 1., 2001, São Pedro. Anais... São Pedro: Centro de Tecnologia de Carnes, 2001. p.123-142.

MOURA, A.C. et al. Efeitos da injeção de cloreto de cálcio pósmorte e tempo de maturação no amaciamento e nas perdas por cozimento do músculo Longissimus dorsi de animais Bos indicus e Bos taurus selecionados para ganho de peso. Revista Brasileira de Zootecnia, Viçosa, v.28, n.6, p.1382-1389, 1999.

OLIVEIRA, I. et al. Postmortem changes and tenderness of Santa Inês as influence by age. In: INTERNATIONAL CONGRESS OF MEAT SCIENCE AND TECHNOLOGY, 49., 2003, Campinas. Anais... Campinas: Centro de Tecnologia de Carnes, 2003. p.197.

OSÓRIO, J.C.S. et al. Métodos para avaliação da produção de carne ovina: in vivo, na carcaça e na carne. Pelotas: UFPEL, 1998. 107p.

PARDI, M.C. et al. Ciência, higiene e tecnologia da carne. Goiânia: UFG, 2001. 623p.

PEREZ, J.R.O. et al. Efeito dos dejetos de suíno na qualidade da carne de ovino. In: REUNIÃO DA SOCIEDADE BRASILEIRA
DE ZOOTECNIA, 34., 1997, Juiz de Fora. Anais... Juiz de Fora, Sociedade Brasileira de Zootecnia, 1997. p.391.

PRATES, J.A.M. Maturação da carne dos mamíferos: 1. Caracterização geral e modificações físicas. Revista Portuguesa de Ciências Veterinárias, Lisboa, v.95, n.533, p.34-41, 2000.

ROTA, E.L. Efeito da castração e idade de abate sobre a qualidade da carne de cordeiros da raça Corriedale. 2005. 87f. Tese (Doutorado em Zootecnia) - Faculdade de Agronomia Eliseu Maciel, Universidade Federal de Pelotas, Pelotas.

WHEELER, T.L. et al. Effects of calcium chloride injection and hot boning on the tenderness of round muscles. Journal of Animal Science, Champaign, v.69, n.12, p.4871-4875, 1991.

SAÑUDO, C. Factors affecting carcass and meat quality in lambs. In: REUNIÃO ANUAL DA SOCIEDADE BRASILEIRA DE ZOOTECNIA, 39., 2002, Recife. Anais... Recife: Sociedade Brasileira de Zootecnia, 2002. p.434-455.

SAS. User's guide: stat, version, 6.12. 4.ed. Cary: SAS Institute, 1996. V.1/2.

ZAPATA, J.F.F. et al. Physical and functional characteristics of tropical lamb aged for 21 days. In: INTERNATIONAL CONGRESS OF MEAT SCIENCE AND TECHNOLOGY, 49., 2003, Campinas. Anais... Campinas: Centro de Tecnologia de Carnes, 2003. p.195-196. 